\title{
Syndrome Differentiation in Chinese Herbal Medicine for Irritable Bowel Syndrome: A Literature Review of Randomized Trials
}

\author{
Qing Li, Guo-Yan Yang, and Jian-Ping Liu \\ Center for Evidence-Based Chinese Medicine, Beijing University of Chinese Medicine, Beijing 100029, China
}

Correspondence should be addressed to Jian-Ping Liu; jianping_l@hotmail.com

Received 6 January 2013; Accepted 6 February 2013

Academic Editor: Aiping Lu

Copyright (C) 2013 Qing Li et al. This is an open access article distributed under the Creative Commons Attribution License, which permits unrestricted use, distribution, and reproduction in any medium, provided the original work is properly cited.

\begin{abstract}
Traditional Chinese medicine (TCM) has been commonly used for irritable bowel syndrome (IBS). Syndrome differentiation is one of the important characteristics of TCM. To assess the application and basic characteristics of syndrome differentiation in randomized controlled trials (RCTs) of Chinese herbal medicine for IBS, we performed this paper. We conducted electronic searches in main Chinese and English databases till March 2012. A total of 735 RCTs involving 67,784 IBS participants were included. 224 (30.5\%) studies applied syndrome differentiation. The major syndromes of IBS patients were the syndrome of liver stagnation and spleen deficiency (56.8\%), spleen-stomach weakness (49.4\%), spleen-kidney yang deficiency (48.1\%), and cold and heat in complexity (29.6\%). Herbal formulas were prescribed based on syndrome differentiation in 202 studies. Chinese patent medicine was more commonly used in studies that only enrolled patients with a specific syndrome. 15 studies compared the therapeutic effect among different syndromes, of which 6 studies showed that there were significant differences among different syndromes. The low use of TCM syndrome differentiation in randomized trials of Chinese herbal medicine for IBS results in the poor pertinence of treatment. TCM syndrome differentiation should be used in further studies at the stage of recruitment, treatment, and data analyses.
\end{abstract}

\section{Introduction}

Irritable bowel syndrome (IBS) is a common functional gastrointestinal disorder characterized by chronic or recurrent symptoms attributed to the intestines including abdominal pain, bloating, and alternations in bowel habits. There are no obvious structural abnormalities, but the whole intestine has an excessive or abnormal response to the stimulation. Epidemiologic studies $[1,2]$ indicated that the prevalence of IBS in Western and Asian countries was from 15\% to $24 \%$ and from $5 \%$ to $10 \%$, respectively. Currently, though standard medical treatment such as antidepressants, antispasmodics, 5-HT3 receptor antagonists, 5-HT4 agonists, and probiotics was commonly applied for IBS, the effect is still unsatisfactory. Chinese herbal medicine has been widely used in the treatment of IBS, and some systematic reviews and meta-analysis [3-5] showed that herbal medicine had a great potential benefit in treating IBS.
According to TCM theory, treatment based on syndrome differentiation ("bian zheng lun zhi" in Chinese), is the basic principle of identifying and treating disease in TCM. Syndrome (Zheng) is a presentation of the pathological changes of a certain disease course, revealing the location, cause and nature of a disease, the correlation between pathogenic factors and healthy factors, and the body's ability to resist a disease. The aim of syndrome differentiation is based on data collected from four diagnostic methods (inspection, listening and smelling, inquiry, and palpation), symptoms, and signs to reveal the location and nature of the disease and provide the best treatment for patients [6]. Therefore, syndrome differentiation is precondition and fundamental for treatment. Since disease is a dynamic process, there may be different syndromes in different phases of a disease. So the exact syndrome differentiation can help doctors to select right herbal formula to provide the best treatment for patients in certain phases of a disease. 
It is believed that individualized, syndrome differentiation-based TCM treatment would be more effective than nondifferentiation-based TCM treatment [7]. However, previous systematic reviews or meta-analysis $[8,9]$ published regarding the effectiveness of Chinese herbal medicine for IBS paid little attention to the application of syndrome differentiation. We searched the published or unpublished randomized controlled trials of Chinese herbal medicine for the treatment of IBS based on a Cochrane systematic review, assessed the application of syndrome differentiation among these randomized controlled trials, and analyzed the basic characteristics of studies which applied the syndrome differentiation, in order to provide the basis for further studies regarding Chinese herbal medicine based on syndrome differentiation for IBS.

\section{Materials and Methods}

2.1. Source of the Literature and Search Strategy. The following electronic databases were searched, regardless of publication status: the Chinese National Knowledge Infrastructure Database (CNKI) (1979-2012), the Chinese Science and Technology Periodical Database (VIP) (1989-2012), the Chinese Biomedical Database (CBM) (1978-2012), the Wanfang Database (1985-2011), PubMed (1966-2012), and the Cochrane Library (Issue 1, 2012). All the searches ended in March 2012. The search terms included "chang_yi_ji_zong_he_zheng" (irritable bowel syndrome), "zhong_yi" (Chinese medicine), "zhong_yao" (Chinese herbs), "zhong_yi_yao" (traditional Chinese medicine), "zhong_cheng_yao" (Chinese patent medicine), "zhong_cao_yao" (Chinese herb medicine), "zhong-

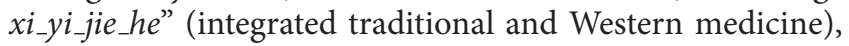
"sui_ji" (randomized), "dui_zhao" (controlled), "irritable bowel syndrome," "herb," "Chinese medicine," "traditional medicine," "plant extracts," "alternative medicine," "complementary medicine," "randomized controlled trial," and "controlled clinical trial."

2.2. Inclusion Criteria. Studies meeting the following four criteria were included. (1) Randomized controlled trials, regardless of publication status; (2) participants with IBS, regardless of age, sex, ethnic origin, and clinical type (diarrhea or constipation predominated); (3) Chinese herbal medicine, regardless of formulation, preparation, dosage, or delivery. The control intervention could be no treatment, placebo, or conventional medicine. Co-intervention was also allowed if they were applied in all arms; (4) clinical outcomes included symptoms, quality of life, recurrence, number, and type of adverse events.

2.3. Exclusion Criteria. The following studies were excluded. (1) Duplication: the same data of patients with the same authors published in different journals; (2) information of participants, interventions, or outcomes were not available.

2.4. Study Selection and Data Extraction. Two authors (Q. $\mathrm{Li}$ and G.-Y. Yang) selected studies independently according to the inclusion and exclusion criteria. We used Epidata 3.1 software to develop a predesigned data extraction form, and two authors (Q. Li and G.-Y. Yang) extracted data independently. The following data were extracted: study design and setting, clinical type of IBS, diagnostic criteria, criteria of syndrome differentiation, inclusion and exclusion criteria, herbal medicine (name of herbs, preparation, means of delivery, and duration of intervention), control, outcome measures, and number and type of adverse events.

2.5. Risk of Bias Assessment. The following items were independently assessed by two authors (Q. Li and G.-Y. Yang) using the Cochrane risk of bias tool recommended by the Cochrane Reviewer's Handbook 5.0.2 [10]: random sequence generation, allocation concealment, blinding, completeness of outcome data, selective outcome reporting, and other potential source of bias. The risk of bias for each item was graded as "low risk," "unclear risk," or "high risk." Disagreements were submitted to J.-P. Liu to resolve.

2.6. Data Analysis. Data were presented as frequency or percentage. $t$-test or Wilcoxon rank sum test was used for the comparisons of quantitative data between groups, while $\chi^{2}$ test was used for the comparisons of qualitative data between groups. SPSS 17.0 software was used for all the data analysis. $P<0.05$ was considered to be statistically significant.

\section{Results}

3.1. Literature Research and Study Selection. Our initial searches identified 6,382 references: 6,141 from Chinese databases and 241 from English databases. After study selection, a total of 735 randomized controlled trials were included (Figure 1).

3.2. Description of Included Studies. In total, $735 \mathrm{RCT}$ involving 67,784 IBS patients were included in this paper. The average sample size was 92 patients (ranging from 20 to 360). The majority of studies were carried out in China (724/735, 98.5\%). $383(383 / 735,52.1 \%)$ studies enrolled IBS patients with diarrhea-predominant type, 80 (80/735, 10.9\%) studies enrolled those with constipation-predominant type, $6(6 / 735$, $0.8 \%)$ studies enrolled both types of IBS, and 266 (266/735, $36.2 \%)$ studies did not specify the type of IBS in their patients. $488(488 / 735,66.4 \%)$ studies used Rome criteria or Manning criteria for the diagnosis of IBS, $174(174 / 735$, $23.7 \%)$ studies used Chinese national criteria, 24 (24/735, $3.3 \%)$ studies used self-defined diagnostic criteria mainly based on Rome criteria, and the remaining 49 (49/735, 6.7\%) studies did not specify the diagnostic criteria. The majority of studies $(584 / 735,80.8 \%)$ used herbal decoction. The control intervention was placebo in $21(21 / 735,2.9 \%)$ studies, no intervention in one $(1 / 735,0.1 \%)$ study, and conventional medicine in $534(534 / 735,72.7 \%)$ studies. 179 (179/735, $24.4 \%)$ studies compared herbal medicine combined with conventional medicine versus conventional medicine alone. All studies reported outcome of the IBS related symptoms, $24(24 / 735,3.3 \%)$ studies reported quality of life, 97 (97/735, 


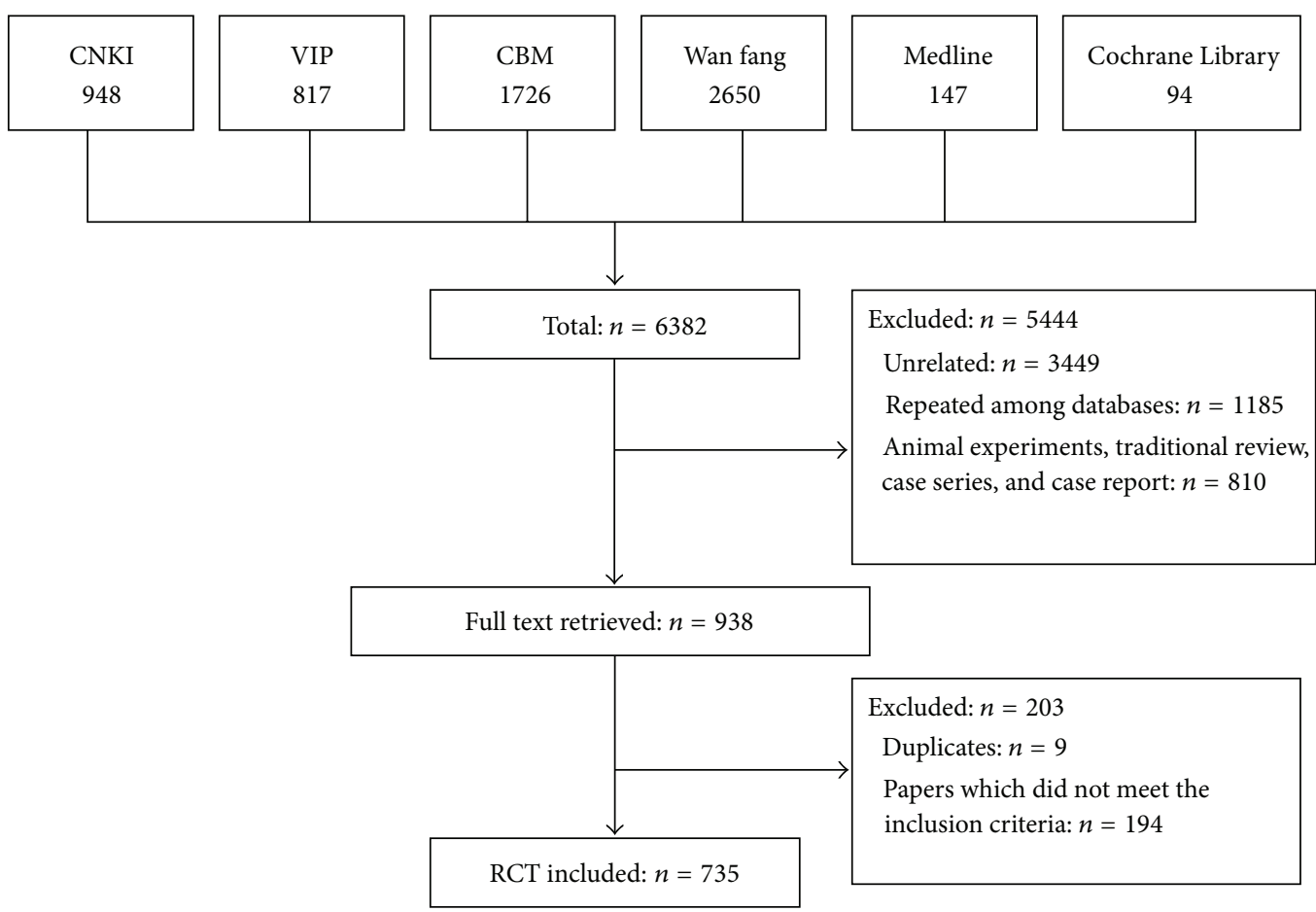

FIGURE 1: Flowchart of the literature search and study selection.

13.2\%) studies reported relapse, and 193 (193/735, 26.3\%) studies reported outcome of adverse events.

3.3. Risk of Bias in Included Studies. Of the 735 included studies, $79(79 / 735,10.7 \%)$ specified the methods for generation of random sequence. Among them, 53 (53/79, 67.1\%) studies used random number table or computer-generated numbers, $17(17 / 79,21.5 \%)$ studies used statistical software such as SAS or SPSS, eight $(8 / 79,10.1 \%)$ studies used drawing lots, and one $(1 / 79,1.3 \%)$ study used coin tossing. Ten $(10 / 735,1.4 \%)$ studies provided information about allocation concealment. Among them, eight (8/10, 80.0\%) studies used sealed envelope, and two $(2 / 10,20.0 \%)$ studies used central control for the allocated treatment. Blindings were reported in $25(25 / 735,3.4 \%)$ studies. Among them, 14 (14/25, 56.0\%) studies reported double blinding, and $11(11 / 25,44.0 \%)$ studies reported single blinding. 25 (25/735, 3.4\%) studies reported the information of withdrawal and dropout, and $12(12 / 25,48.0 \%)$ studies applied intention-to-treat analysis. $66(66 / 735,9.0 \%)$ studies were judged with high risk as some prespecified outcomes were not reported in the results. $11(11 / 735,1.5 \%)$ studies reported a pretrial estimation of sample size.

In conclusion, the general methodological quality of most included trials was unclear or high risk of bias.

3.4. Application Condition of Syndrome Differentiation in Included Studies. Of the 735 studies, 224 (224/735, 30.5\%) applied syndrome differentiation and reported the related data. Although $11(11 / 735,1.5 \%)$ studies mentioned syndrome differentiation in the diagnostic criteria of IBS, they did not report the results of syndrome differentiation. Therefore, in this study, we mainly analyzed the 224 studies that both applied syndrome differentiations and reported the related data.

Among the 224 studies, 153 (153/224, 68.3\%) studies specified the diagnostic criteria for TCM syndrome differentiation, and following was the most commonly used criteria: Clinical Research Guidelines for New Chinese Herbal Drug [11], Chinese Internal Medicine, and the Diagnosis and Treatment Protocol of Integrated Chinese and Western Medicine for Irritable Bowel Syndrome (draft) [12]. There were two types of syndrome differentiation, $143(143 / 224$, $63.8 \%$ ) studies conducted syndrome differentiation prior to inclusion of participants, and a specified TCM syndrome was one of their inclusion criteria. These studies only enrolled patients with a specified TCM syndrome. The remaining 81 $(81 / 224,36.2 \%)$ studies conducted syndrome differentiation after inclusion of participants, and their inclusion criteria did not involve certain TCM syndrome(s). In addition, the majority of studies $(166 / 224,74.1 \%)$ performed syndrome differentiation for all patients in both groups, while the other 58 studies $(58 / 224,25.9 \%)$ performed it only for patients in herbal group. 202 studies $(202 / 224,90.2 \%)$ selected herbal medicine based on relevant results of syndrome differentiation, while the other $22(22 / 224,9.8 \%)$ studies though applied syndrome differentiation, did not select herbal medicine based on the syndrome differentiation.

3.5. TCM Syndrome Distribution in Patients with IBS. Of the 224 studies, $143(143 / 224,63.8 \%)$ studies enrolling patients 
with a specific TCM syndrome were excluded because they might fail to represent an authentic overview of syndrome distribution among IBS patients. The remaining $81(81 / 224$, $36.2 \%)$ studies were included for analysis of TCM syndrome distribution.

A total of 48 TCM syndromes were identified from the 81 studies. The most commonly reported syndromes were the syndrome of liver stagnation and spleen deficiency $(46 / 81$, $56.8 \%)$, spleen-stomach weakness (40/81, 49.4\%), spleenkidney yang deficiency $(39 / 81,48.1 \%)$, and cold and heat in complexity (14/81, 29.6\%). 40 of 81 studies (40/81, 49.4\%) enrolled patients with diarrhea-predominant IBS, three studies enrolled patients with constipation-predominant IBS, one study enrolled patients with mixture of both types, and the remaining studies did not specify the type of IBS. Considering the few papers reporting constipation-predominant IBS and the mixture of both types of IBS, the current paper only analyzed the data of syndrome distribution in IBS patients with diarrhea-predominant type. For IBS patients with diarrheapredominant type, the most commonly seen syndrome was spleen-stomach weakness $(23 / 40,57.5 \%)$, followed by spleenkidney yang deficiency $(21 / 40,52.5 \%)$, liver stagnation and spleen deficiency $(21 / 40,52.5 \%)$, cold and heat in complexity $(11 / 40,27.5 \%)$, and spleen qi deficiency $(9 / 40,22.5 \%)$.

3.6. Medication Characteristics of 224 Studies Applying Syndrome Differentiation. For preparation, 190 (190/224, 84.8\%) studies used herbal decoction, of which 77 studies used standardized Chinese herbal formula, while the other 113 studies used modified formula according to different syndromes. Compared with studies that enrolled patients with different TCM syndromes, we found that studies that enrolled patients with a specific TCM syndrome more frequently used Chinese patent medicine. We also found that studies that enrolled patients with different TCM syndromes were more likely to use modified herbal formula (Table 1).

3.7. Application of Syndrome Differentiation in Therapeutic Effect Evaluation. Among the 81 studies enrolling patients with different TCM syndromes, 15 (15/81, 18.5\%) studies considered the difference of syndrome in therapeutic effect evaluation. Among these 15 studies, 13 (13/15, 86.7\%) compared effect among different TCM syndromes in herbal group, of which $6(6 / 13,46.2 \%)$ studies showed that there were significant differences among different syndromes. $2(2 / 15$, $13.3 \%)$ studies not only compared effect among different TCM syndromes within each group, but also conducted analysis among groups and found that there were no significant differences between groups for each syndrome (Table 2).

\section{Discussion}

In TCM clinical practice, practitioners prescribed herbal formula according to syndrome type identified through inspection, smelling, requesting, and pulse taking. Applying TCM syndrome differentiation in the clinical trial may reflect the practice and inform the prescription of the treatment. Therefore, we believe that randomized trials of Chinese herbal medicine should be able to embody the special feature of TCM syndrome differentiation under the premise of ensuring high methodological quality. Among 735 randomized trials included in this paper, only a small proportion of studies applied syndrome differentiation and selected herbal formula based on syndrome differentiation. The majority of studies failed to apply syndrome differentiation, which was diverse from TCM theory and practice. We recommend two ways to conduct syndrome differentiation in TCM clinical studies: first, apply syndrome differentiation in recruitment by using certain syndrome as one of the inclusion criteria to limit subgroup populations; second, apply syndrome differentiation after the recruitment, and in therapeutic effect evaluation, make subgroup analyses based on different syndromes. If individualized herbal treatment is used, then modified herbal formula should be prescribed based on different syndromes.

In this paper, we found that the most common syndromes of IBS patients were the syndrome of liver stagnation with spleen deficiency, weakness of spleen and stomach, deficiency of spleen and kidney yang, and cold and heat in complexity. In patients with diarrhea-predominant IBS, the syndromes of weakness of spleen and stomach, deficiency of spleen and kidney yang, liver depression with spleen deficiency, cold and heat in complexity, and spleen qi deficiency are the more prevalent ones, which was consistent with the results of previous studies [13-15].

Chinese patent medicine was more frequently used in studies enrolling patients with specified syndrome, while modified decoction according to syndromes was more frequently used in studies enrolling IBS patients regardless of syndrome type. Chinese patent medicine is made from Chinese materia medica according to standardized TCM prescription, with fixed components and proportion of drugs dose. Therefore, it is different from decoction which is so flexible that can be modified based on different syndromes, and its application is only for patients with a specified TCM syndrome. It explains why Chinese patent medicine is used more frequently in studies enrolling IBS patients with a specified syndrome.

Syndrome differentiation is the most distinctive character of TCM practice. It is believed that TCM treatment based on syndrome differentiation would be more effective; however, few studies demonstrate additional benefit from TCM syndrome differentiation. Among 735 studies included in this paper, only one trial [16] published in English compared the therapeutic effect of individualized treatment based on syndrome differentiation with that of standard Chinese herbal prescription and found that the therapeutic effect of individualized treatment based on syndrome differentiation was more sustainable in treating IBS. This study was published in 1998, and since then no other studies were published to verify this finding. Therefore, more large well-designed studies using syndrome differentiation are warranted to confirm if any additional therapeutic benefits can be achieved by using syndrome differentiation.

Although TCM syndrome differentiation was applied in some randomized controlled trials of Chinese herbal medicine for IBS, we found there were some problems in application. 
TABLE 1: Comparison of medication characteristics between two types of syndrome differentiation in randomized trials.

\begin{tabular}{|c|c|c|c|c|}
\hline Item & $\begin{array}{l}\text { Studies that enrolled patients } \\
\text { with a specific TCM syndrome } \\
\text { only } \\
(n=143)\end{array}$ & $\begin{array}{l}\text { Studies that enrolled patients } \\
\text { with different TCM syndromes } \\
\qquad(n=81)\end{array}$ & Statistics & $P$ value \\
\hline \multicolumn{5}{|l|}{ Preparation ${ }^{*}$} \\
\hline Decoction & $113(79.0 \%)$ & 77 (95.1\%) & \multirow{3}{*}{-} & \multirow{3}{*}{0.001} \\
\hline Chinese patent medicine & $12(8.4 \%)$ & $0(0.0 \%)$ & & \\
\hline Hospital preparation & $18(12.6 \%)$ & $4(4.9 \%)$ & & \\
\hline \multicolumn{5}{|l|}{ Modified } \\
\hline Yes & $52(46.0 \%)$ & $61(79.2 \%)$ & \multirow{2}{*}{$\chi^{2}=20.946$} & \multirow{2}{*}{$<0.001$} \\
\hline No & $61(54.0 \%)$ & $16(20.8 \%)$ & & \\
\hline \multicolumn{5}{|l|}{ Route of delivery } \\
\hline Oral & $139(97.2 \%)$ & $74(91.4 \%)$ & \multirow{2}{*}{$\chi^{2}=2.635$} & \multirow[t]{2}{*}{0.105} \\
\hline Other & $4(2.8 \%)$ & $7(8.6 \%)$ & & \\
\hline Course of treatment (days) & $31 \pm 14$ & $30 \pm 10$ & $Z=-1.645$ & 0.100 \\
\hline Time of followup (days) & $121 \pm 83$ & $150 \pm 53$ & $Z=-0.176$ & 0.860 \\
\hline
\end{tabular}

${ }^{*}$ Fisher's exact test was used in statistical analysis.

(1) The criteria of TCM syndrome differentiation were not clear or consistent. Among 224 studies which applied syndrome differentiation, 71 studies (31.6\%) did not specify the criteria for syndrome differentiation. Since syndrome differentiation is an important part of diagnosis and treatment for patients, it is essential to use reliable and practical criteria to ensure the accuracy, consistency, and repeatability of syndrome differentiation.

(2) Chinese herbal formula was not prescribed based on the syndrome differentiation. Although 22 of 224 studies applied syndrome differentiation in recruitment stage, in treatment stage patients with different syndromes were given the same herbal formula, which violated the basic principle of TCM syndrome differentiation and might affect the therapeutic effect of herbal medicine. Practitioners should make prescriptions corresponding to syndromes of patients, no matter using standard prescription or modified herbal formula. It is believed that only selecting herbal formula based on TCM syndrome differentiation could ensure clinical efficacy.

(3) The methods for effect analysis were inadequate. In this paper, we found no studies used stratified randomization, and only 15 studies considered the influence of syndrome on effect in statistical analysis, of which 2 used stratification analysis, 4 analyzed the difference of effect among patients with different syndromes that received the same prescription, and 9 analyzed the difference of effect among patients with different syndromes that received different prescriptions. The majority of studies neglected syndromes' influence on effect resulting in unreliable findings and limited the value of clinical application. Since the reaction of patients with different syndromes to the same prescription is different, outcomes will also be different. Considering the influence of syndromes on effect, during recruitment stage of a randomized controlled trial, patients should be stratified according to different syndromes before randomization, or during statistical analysis stage, and perform stratification analysis to ensure objective evaluation of effect.

(4) Syndrome differentiation was just performed in treatment group and not in control group. Among 224 studies using syndrome differentiation, a large number of the studies did not perform syndrome differentiation in control group. Only when syndrome differentiation is performed in both groups, the baseline information can be available about syndrome differentiation of two groups, which is very important for stratified randomization or data analysis to control the influence of syndrome on effect.

To sum up, the application of syndrome differentiation in the treatment of irritable bowel syndrome is poor. In most of the studies, the selection of Chinese prescription is not based on syndrome differentiation, which might affect the pertinence of treatment of Chinese herbs. What is worse there are a series of problems in application of syndrome differentiation. More multicentered large-scale randomized controlled trials performing appropriate TCM syndrome differentiation are warranted. We make the following recommendations for further studies. (1) Stratified randomization. All participants should be stratified according to their syndromes before randomization. It is also suggested to use a specific syndrome as one of the inclusion criteria, and only enroll patients with that specific syndrome. (2) Perform syndrome differentiation for all participants, regardless of the intervention they received. Specially, definite criteria of TCM syndrome differentiation should be reported. (3) Prescribe herbal treatment based on syndrome differentiation. (4) Report diseaserelated outcomes, such as endpoint, quality of life, or global improvement of symptoms through validated instrument and 
TABLE 2: Presentations of therapeutic effect of herbal medicine in relation to TCM syndromes among 15 RCTs.

\begin{tabular}{|c|c|c|c|c|}
\hline Study ID & TCM syndrome & Herbal formula & Control & Main findings \\
\hline Cheng WJ 2000* & $\begin{array}{l}\text { Qi stagnation and blood } \\
\text { stasis } \\
\text { Spleen and stomach } \\
\text { weakness } \\
\text { Spleen-kidney yang } \\
\text { deficiency } \\
\text { Other syndromes }\end{array}$ & Modified Li Zhong Tang & $\begin{array}{l}\text { Sodium } \\
\text { cromoglycate, } \\
\text { diazepam, } \\
\text { vitamin B1 } \\
\text { plus loperamide } \\
\text { hydrochloride if } \\
\text { diarrhea exists }\end{array}$ & $\begin{array}{l}\text { Herbal medicine showed a statistically } \\
\text { significant benefit for short-term and } \\
\text { long-term effects compared with } \\
\text { conventional medicine } \\
\text { No significant difference among } \\
\text { patients with different TCM } \\
\text { syndromes for short-term and } \\
\text { long-term effects in herbal group }\end{array}$ \\
\hline Hu ZL $2000^{*}$ & $\begin{array}{l}\text { Liver stagnation and spleen } \\
\text { deficiency } \\
\text { Liver-stomach disharmony } \\
\text { Spleen-stomach } \\
\text { deficiency-cold }\end{array}$ & Jianwei Yuyang Pill & $\begin{array}{l}\text { Oryzanol } \\
\text { bifidobacterium } \\
\text { triple viable } \\
\text { capsules }\end{array}$ & $\begin{array}{l}\text { Herbal medicine showed a statistically } \\
\text { significant benefit for short-term and } \\
\text { long-term effects compared with } \\
\text { conventional Western medicine } \\
\text { therapy } \\
\text { In herbal group, there was no } \\
\text { significant difference between patients } \\
\text { with syndrome of liver depression and } \\
\text { spleen insufficiency and syndrome of } \\
\text { liver-stomach disharmony for } \\
\text { short-term effect, but they all were } \\
\text { significantly better than patients with } \\
\text { syndrome of spleen-stomach } \\
\text { deficiency-cold }\end{array}$ \\
\hline
\end{tabular}

Modified Xiao Yao San and Si Jun Zi Tang used orally; Lian Qin Tang used by retention enema

Xiangsha Weiling Tang plus

Liver-stomach disharmony Pu Huang used orally; Spleen deficiency with Lian Qin Tang used by excessive damp

Duan GY 2002* Spleen-kidney yang deficiency

Yin-deficiency and congealing cold retention enema

Li Zhong Tang and Si Shen Wan plus Yuan $\mathrm{Hu}$ and $\mathrm{Fu}$ Pian used orally; Lian Qin Tang used by retention enema Wen Pi Tang plus Dang Gui, Chuan Xiong, and Shu Di Huang used orally Lian Qin Tang used by retention enema
Liver qi attacking the spleen Modified Si Ni San plus Tong Weakness of spleen and

Tian JY $2002^{*}$ stomach Spleen-kidney yang deficiency
Oryzanol and bifidobacterium viable capsule
The total effective rate in herbal group was significantly higher than in control group

In herbal group, the total effective rate in patients with syndrome of

liver-spleen disharmony was the best, followed by patients with syndrome of spleen insufficiency with damp harassment, spleen-kidney yang deficiency, yin-deficiency and pathogenic cold

\section{Xie Yao Fang}

Modified Shenling Baizhu San

Li Zhong Wan plus Si Shen Wan
Western

medicine based on symptoms
Herbal medicine showed a statistically significant benefit for clinical effect compared with western medicine based on symptoms There was no significant difference among patients with different TCM syndromes for clinical effect in herbal group

Herbal medicine showed a statistically significant benefit for clinical effect Modified Tong Xie Yao Fang Liver stagnation and spleen deficiency

Weakness of spleen and stomach Spleen-kidney yang deficiency no. 1 combined with Shi Yi Fang

Modified Tong Xie Yao Fang No. 2 combined with Shi Yi Fang Modified Tong Xie Yao Fang No. 3 combined with Shi Yi Fang
Pinaverium bromide plus fluoxetine if psychiatric symptoms existed, plus alprazolam if sleep disorders existed compared with conventional western medicine therapy In herbal group, clinical effect on patients with syndrome of liver depression with spleen insufficiency was the best, followed by patients with syndrome of weakness of spleen and stomach, and syndrome of spleen-kidney yang deficiency 
TABle 2: Continued.

\begin{tabular}{|c|c|c|c|}
\hline Study ID & TCM syndrome & Herbal formula & Control \\
\hline Li QH 2006* & $\begin{array}{l}\text { Liver stagnation and spleen } \\
\text { deficiency } \\
\text { Weakness of spleen and } \\
\text { stomach } \\
\text { Spleen-kidney yang } \\
\text { deficiency }\end{array}$ & $\begin{array}{l}\text { Modified Tong Xie Yao Fang } \\
\text { no. } 1 \\
\text { Modified Tong Xie Yao Fang } \\
\text { no. } 2 \\
\text { Modified Tong Xie Yao Fang } \\
\text { no. } 3\end{array}$ & $\begin{array}{l}\text { Pinaverium } \\
\text { bromide plus } \\
\text { belladonna if } \\
\text { abdominal pain } \\
\text { exists, plus } \\
\text { compound } \\
\text { diphenoxylate if } \\
\text { diarrhea exists, } \\
\text { plus doxepin if } \\
\text { psychiatric } \\
\text { symptoms } \\
\text { existed }\end{array}$ \\
\hline
\end{tabular}

Spleen-stomach deficiency-cold

Liu AQ 2006* Cold-damp stagnation in large intestine Stagnant of qi movement
Modified Xiangsha Liujunzi

Tang

Modified Wei Ling Tang

Modified Liu Mo Tang
Western medicine based on symptoms

Main findings

Herbal medicine showed a statistically significant benefit for clinical effect compared with conventional Western medicine therapy

In herbal group, there was no significant difference between patients with syndrome of liver depression and spleen insufficiency and syndrome of weakness of spleen and stomach, but they all were significantly better than patients with syndrome of spleen-kidney yang deficiency

Stratified analysis according to syndrome differentiation The total effective rate in herbal group was significantly higher than control group for patients with syndrome of spleen-stomach deficiency-cold The total effective rate in herbal group was significantly higher than control group for patients with syndrome of cold-damp stagnation in intestines The total effective rate in herbal group was significantly higher than in control group for patients with syndrome of stagnant of qi movement

The total effective rate in herbal group was significantly higher than in control group

no. 1

Modified Tong Xie Yao Fang no. 2

Modified Tong Xie Yao Fang no. 3
Loperamide hydrochloride complexity Weakness of spleen and stomach
There was no significant difference among patients with different TCM syndromes on the total effective rate in herbal group

The total effective rate in herbal group was significantly higher than in control group

no. 1 deficiency

Huang PR 2008* Cold and heat in complexity Weakness of spleen and stomach

Modified Tong Xie Yao Fang no. 2

Modified Tong Xie Yao Fang

Loperamide no. 3

Liver stagnation and spleen deficiency

Cold and heat in complexity

Leng QN 2009* Weakness of spleen and stomach

Food stagnation in stomach and intestines

Spleen-kidney yang deficiency

Modified self-prepared herbal formula no.1

Modified self-prepared herbal formula no. 2

Modified self-prepared herbal formula no. 3

Modified self-prepared herbal formula no. 4

Modified self-prepared herbal formula no. 5

Liver-spleen disharmony Spleen-kidney yang deficiency Spleen qi deficiency
Modified Hu Su Gan San plus Tong Xie Yao Fang Modified Si Shen Wan Modified Shenling Baizhu San hydrochloride

There was no significant difference among patients with different TCM syndromes on the total effective rate in herbal group

The total effective rate in herbal group was significantly higher than in control group

There was no significant difference among patients with different TCM syndromes on the total effective rate in herbal group

The total effective rate in herbal group was significantly higher than in control group

Pinaverium There was no significant difference among patients with different TCM syndromes on the total effective rate in herbal group. 
TABLe 2: Continued.

\begin{tabular}{|c|c|c|c|c|}
\hline Study ID & TCM syndrome & Herbal formula & Control & Main findings \\
\hline He ZM 2010* & $\begin{array}{l}\text { Liver stagnation with } \\
\text { spleen insufficiency } \\
\text { Cold and heat in } \\
\text { complexity } \\
\text { Yin deficiency and } \\
\text { intestines dryness } \\
\text { Intestinal constraint }\end{array}$ & $\begin{array}{l}\text { Modified self-prepared Yiji } \\
\text { Changkang Tang }\end{array}$ & $\begin{array}{l}\text { Western } \\
\text { medicine based } \\
\text { on symptoms }\end{array}$ & $\begin{array}{l}\text { Herbal medicine showed a statistically } \\
\text { significant benefit for clinical effect } \\
\text { compared with conventional western } \\
\text { medicine therapy } \\
\text { There was no significant difference } \\
\text { among patients with different TCM } \\
\text { syndromes for clinical effect in herbal } \\
\text { group }\end{array}$ \\
\hline Li HJ $2010^{* *}$ & $\begin{array}{l}\text { Liver-spleen disharmony } \\
\text { Spleen-kidney yang } \\
\text { deficiency } \\
\text { Cold and heat in } \\
\text { complexity }\end{array}$ & $\begin{array}{l}\text { Modified Chaihu Shugan San } \\
\text { plus Tong Xie Yao Fang } \\
\text { Modified Si Shen Wan plus Li } \\
\text { Zhong Tang } \\
\text { Modified Wu Mei Wan }\end{array}$ & $\begin{array}{l}\text { Western } \\
\text { medicine based } \\
\text { on symptoms }\end{array}$ & $\begin{array}{l}\text { The cure rate in herbal group was } \\
\text { significantly higher than control group } \\
\text { In herbal group, the cure rate in } \\
\text { patients with syndrome of } \\
\text { spleen-kidney yang deficiency was } \\
\text { significantly higher than patients with } \\
\text { liver-spleen disharmony and cold and } \\
\text { heat in complexity, but there was no } \\
\text { significant difference between patients } \\
\text { with syndrome of liver-spleen } \\
\text { disharmony and patients with cold and } \\
\text { heat in complexity }\end{array}$ \\
\hline Bian LQ 2011 ${ }^{* * *}$ & $\begin{array}{l}\text { Liver-spleen disharmony } \\
\text { Weakness of spleen and } \\
\text { stomach } \\
\text { Spleen-kidney yang } \\
\text { deficiency }\end{array}$ & Changan Yihao Fang & Placebo & $\begin{array}{l}\text { Applied stratified analysis according to } \\
\text { syndrome differentiation } \\
\text { The score of IBS-SSS in herbal group } \\
\text { was significantly lower than control } \\
\text { group } \\
\text { There was no significant difference } \\
\text { between two groups for patients with } \\
\text { each syndromes }\end{array}$ \\
\hline Wang C $2012^{*}$ & $\begin{array}{l}\text { Liver qi attacking the spleen } \\
\text { Weakness of spleen and } \\
\text { stomach } \\
\text { Kidney yang deficiency }\end{array}$ & $\begin{array}{l}\text { Tong Xie Yao Fang combined } \\
\text { with trimebutine }\end{array}$ & Trimebutine & $\begin{array}{l}\text { Herbal medicine showed a statistically } \\
\text { significant benefit for clinical effect } \\
\text { compared with trimebutine } \\
\text { In herbal group, patients with } \\
\text { syndrome of liver qi attacking the } \\
\text { spleen showed the best clinical effect, } \\
\text { followed by patients with syndrome of } \\
\text { weakness of spleen and stomach, and } \\
\text { patients with syndrome of kidney yang } \\
\text { deficiency }\end{array}$ \\
\hline
\end{tabular}

${ }^{*}$ represents studies which are taking total effective rate as the primary outcome; ${ }^{* *}$ represents studies which are taking cure rate as the primary outcome; **** represents studies which are taking score of IBS-SSS as the primary outcome.

adverse events. Composite outcomes such as total effective rate should be avoided. (5) Use stratified analysis according to syndrome in therapeutic effect evaluation if there are different syndromes.

\section{Conflict of Interests}

The authors state that there is no conflict of interests in this study.

\section{Authors' Contribution}

Q. Li and G.-Y. Yang contributed equally to this work.

\section{Acknowledgments}

This study was funded by the Program of "Establishing of the Platform for Drug Research and DevelopmentStandard Operation Procedure (SOP) for Clinical Evaluation
Research" of State Center for Drug Research and Development (2011ZX09302-006-01-03). The authors were supported by the Program of Innovative Research Team of Beijing University of Chinese Medicine (2011-CXTD-09).

\section{References}

[1] L. Frank, L. Kleinman, A. Rentz, G. Ciesla, J. J. Kim, and C. Zacker, "Health-related quality of life associated with irritable bowel syndrome: comparison with other chronic diseases," Clinical Therapeutics, vol. 24, no. 4, pp. 675-689, 2002.

[2] O. Grundmann and S. L. Yoon, "Irritable bowel syndrome: epidemiology, diagnosis and treatment: an update for healthcare practitioners," Journal of Gastroenterology and Hepatology, vol. 25, no. 4, pp. 691-699, 2010.

[3] R. Rahimi and M. Abdollahi, "Herbal medicines for the management of irritable bowel syndrome: a comprehensive review," World Journal of Gastroenterology, vol. 18, no. 7, pp. 589-600, 2012. 
[4] S. Dongmei, Z. Shengsheng, L. Jianping, and W. HongBing, "Systematic review of D-IBS treated by Chinese herbal medicine," China Journal of Traditional Chinese Medicne, vol. 24, no. 4, pp. 532-535, 2009.

[5] J. Shi, Y. Tong, J. G. Shen, and H. X. Li, "Effectiveness and safety of herbal medicines in the treatment of irritable bowel syndrome: a systematic review," World Journal of Gastroenterology, vol. 14, no. 3, pp. 454-462, 2008.

[6] Y. C. liu and S. Q. Lei, Basic Theory of Traditional Chinese Medicine, Academic Publishing House, Beijing, China, 2005.

[7] A. P. Lu and K. J. Chen, "Integrative medicine in clinical practice: from pattern differentiation in traditional Chinese medicine to disease treatment," Chinese Journal of Integrative Medicine, vol. 15, no. 2, article 152, 2009.

[8] J. P. Liu, M. Yang, Y. X. Liu, M. L. Wei, and S. Grimsgaard, "Herbal medicines for treatment of irritable bowel syndrome," Cochrane Database of Systematic Reviews, no. 1, Article ID CD004116, 2006.

[9] Z. X. Bian, T. X. Wu, L. Liu et al., "Effectiveness of the Chinese herbal formula Tong Xie Yao Fang for irritable bowel syndrome: a systematic review," Journal of Alternative and Complementary Medicine, vol. 12, no. 4, pp. 401-407, 2006.

[10] J. P. T. Higgins and S. Green, Cochrane Handbook for Systematic Reviews of Interventions Version 5.0.2, The Cochrane Collaboration, 2009.

[11] Z. Xiaoyu, Clinical Research Guidelines for New Chinese Herbal Drug, China Medical Science Press, Beijing, China, 2002.

[12] C. Zhishui, Z. Wandai, W. Beihan et al., "The diagnosis and treatment protocol of integrated Chinese and Western medicine for irritable bowel syndrome (Draft)," Chinese Journal of Integrated Traditional and Western Medicine on Digestion, vol. 13, no. 1, pp. 65-67, 2005.

[13] L. Zhao and Q. Liu, "Research on the TCM syndromes and psychological factors of patients with irritable bowel syndrome," Zhejiang Journal of Traditional ChineseMedicine, vol. 47, no. 7, pp. 475-477, 2012.

[14] B. Wu and S. Zhang, "Progress of syndrome study on diarrheapredominant irritable bowel syndrome (IBS-D)," Beijing Journal of Traditional Chinese Medicine, vol. 26, no. 5, pp. 312-314, 2007.

[15] Digestive Disease Committee of China Society of Integrated Traditional Chinese and Western Medicine, "Consensus and opinion of integrated traditional and western medicine for irritable bowel syndrome," Chinese Journal of Integrated Traditional and Western Medicine, vol. 31, no. 5, pp. 587-590, 2011.

[16] A. Bensoussan, N. J. Talley, M. Hing, R. Menzies, A. Guo, and M. Ngu, "Treatment of irritable bowel syndrome with Chinese herbal medicine: a randomized controlled trial," The Journal of the American Medical Association, vol. 280, no. 18, pp. 15851589, 1998. 


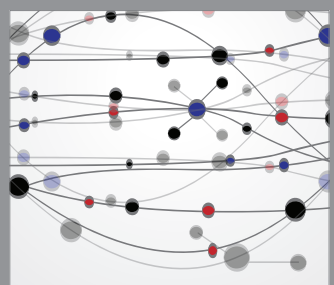

The Scientific World Journal
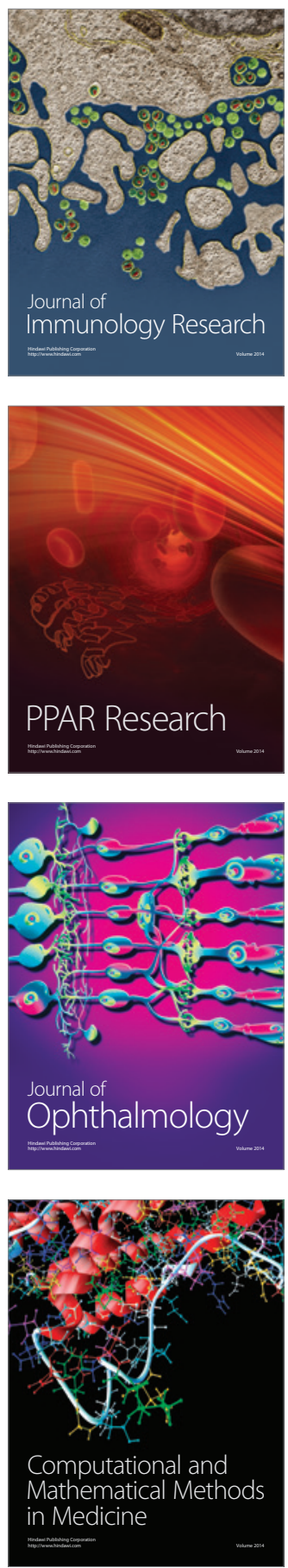

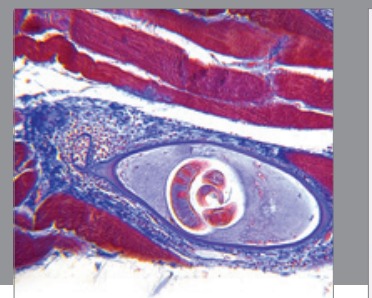

Gastroenterology

Research and Practice
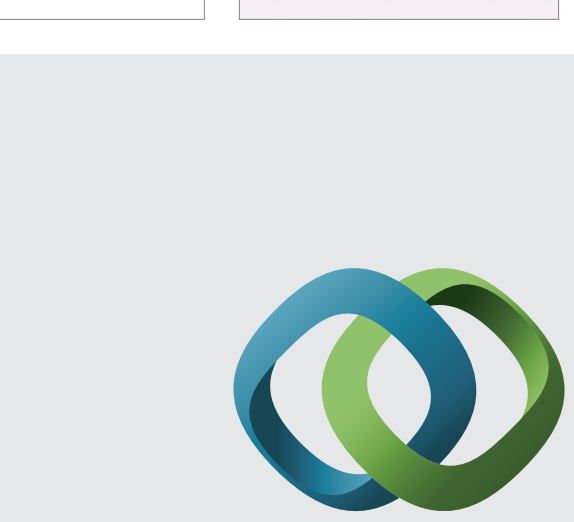

\section{Hindawi}

Submit your manuscripts at

http://www.hindawi.com
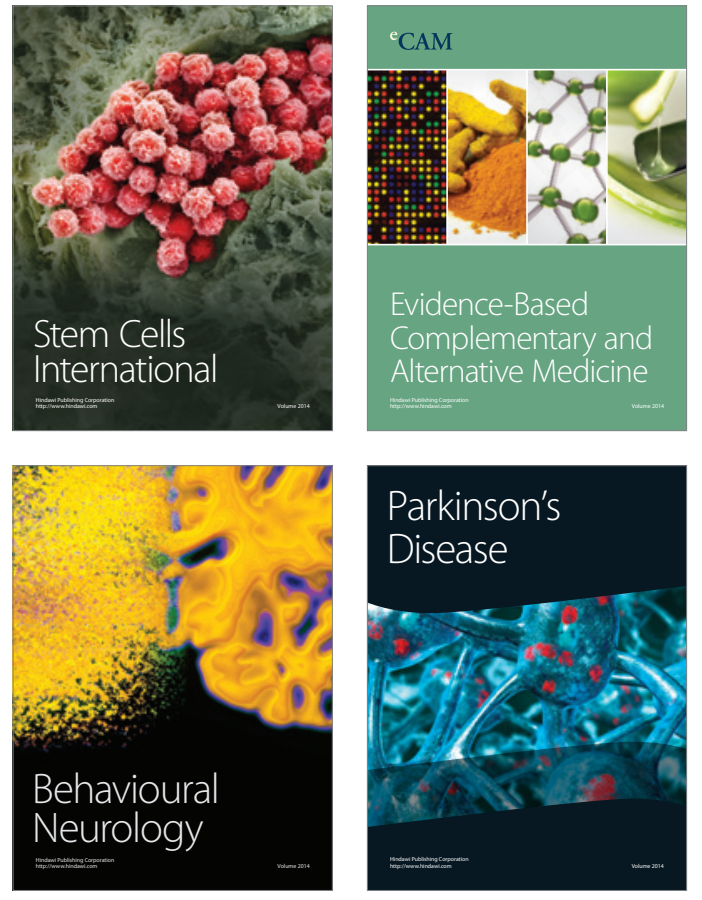
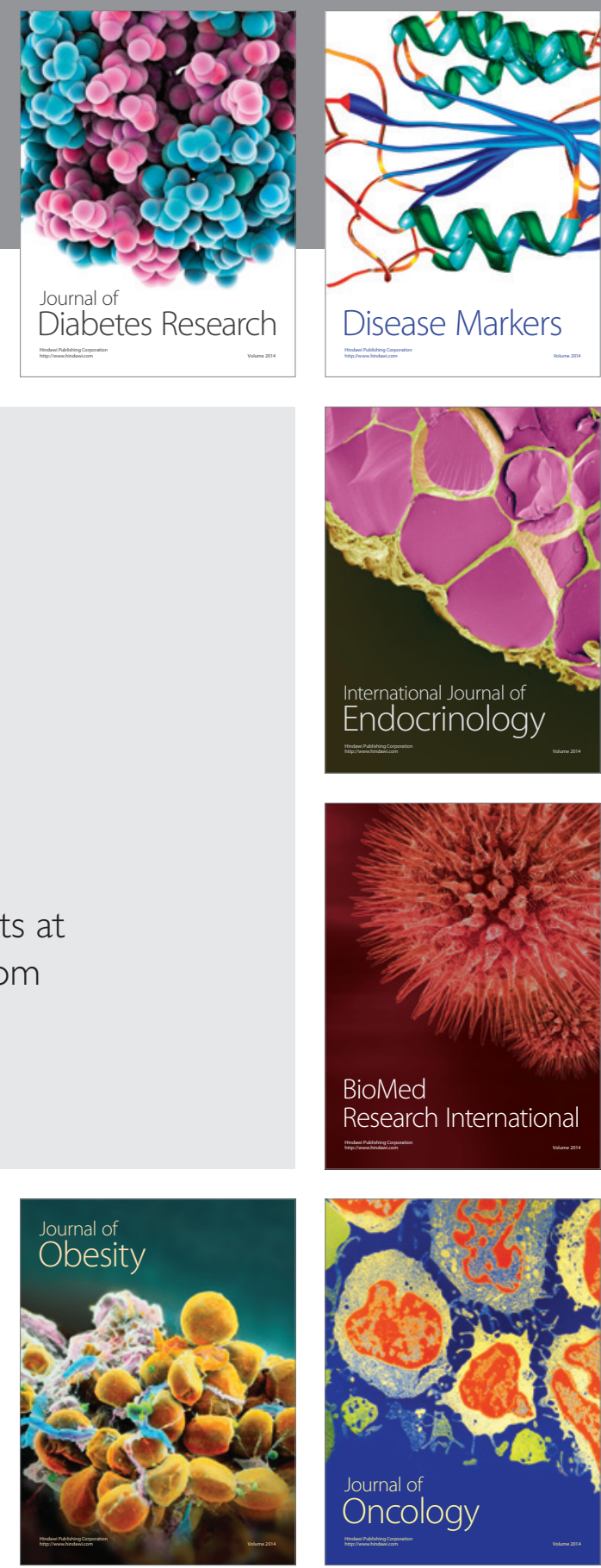

Disease Markers
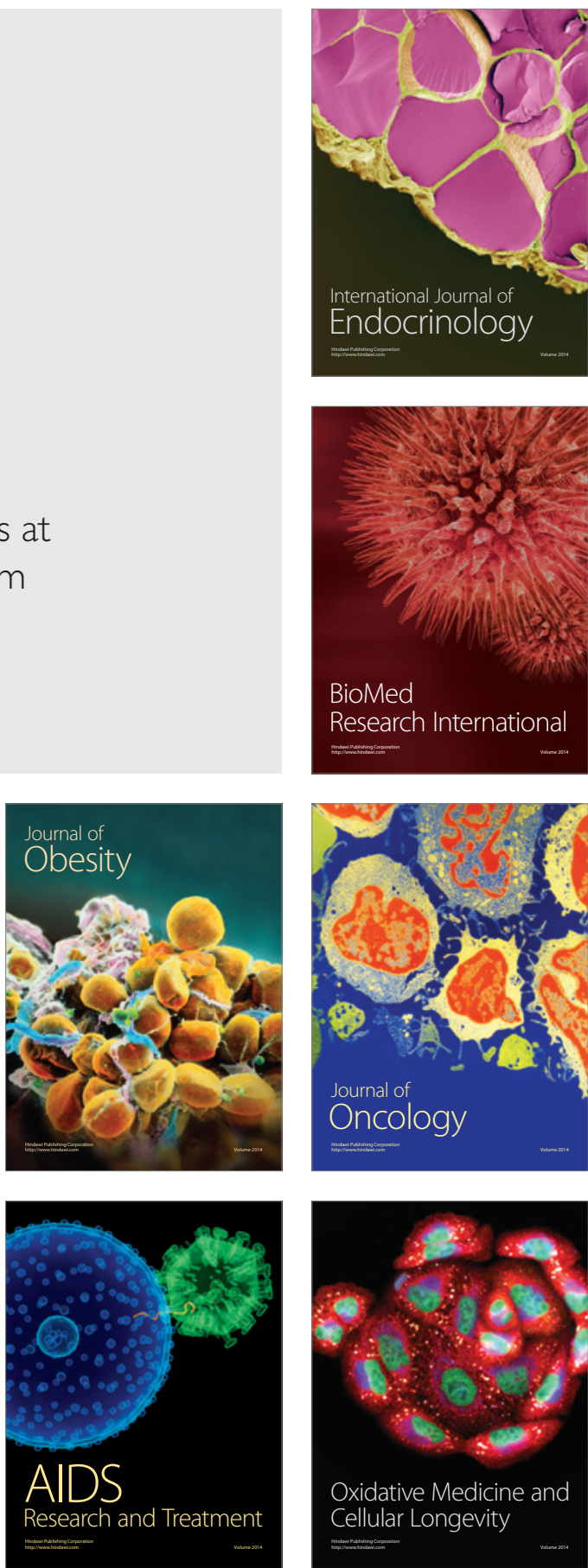